\title{
Volumes of Discrete Groups and Topological Complexity of Homology Spheres
}

\author{
Alexander ReZnikov
}

\begin{abstract}
Can one ascribe a "size" to a discrete group? By that we mean a real-valued invariant, $\mu(\Gamma)$ satisfying the following condition: for any subgroup $\Delta<\Gamma$ of finite index, $\mu(\Delta)=$ $(\Gamma: \Delta) \mu(\Gamma)$. The importance of such an invariant for the structure of $\Gamma$ is clear from the following observation: if $\mu(\Gamma)>0$ and $\Delta_{1}, \Delta_{2}$ are two isomorphic subgroups of $\Gamma$ of finite index, then $\left(\Gamma: \Delta_{1}\right)=\left(\Gamma: \Delta_{2}\right)$.

Some rational-valued invariants satisfying the axiom above, have been studied by Wall [17], Bass [1], Chiswell [7], Brown [4], Stallings [16] etc. and called (generalized) Euler characteristics. These are defined for a FP group $\Gamma$ by $\chi(\Gamma)=\sum(-1)^{i} r k P_{i}$ where $\mathbb{P} \rightarrow \mathbb{Q}$ is a finite f.g. projective resolution of $\mathbb{Q}$ and $r k: K_{0}(\mathbb{Q} \Gamma) \rightarrow \mathbb{Z}$ is a homomorphism and extended to all VFP groups in the usual way [17].

On the other hand, if $\Gamma$ runs through fundamental groups of closed three-manifolds, there are two known invariants, (usually called covering invariants by obvious reason). One is Gromov's simplicial volume, which is positive for any hyperbolic manifold. The other is Wang and Wu's invariant [18], defined for any plumbing of Seifert fibrations.

The approach presented in this paper, takes a new standpoint in dealing the above problem, that is, a "measure-theoretic" one. Namely, we start with "semimultiplicative invariants", analogous to (outer) measure in measure theory and then produce a truly multiplicative invariants by going to lower limit over the directed set of finite index subgroups.

In course of application, we address two fundamental and well-known problems of Gromov and Lyndon:
\end{abstract}

Problem A: (Gromov, see [5]). Consider a category $M_{n}$ of closed manifolds of dimension $n$ with nonzero-degree ways as morphisms. Study a partial order $M \geq N \Leftrightarrow \operatorname{Mor}(M, N) \neq$ $\phi$. For which $N$ the degrees of maps $f: M \rightarrow N$ are bounded for all $M$ ? 
Problem B: (Lyndon, [12], problem 13). Extend and relate the theories of deficiency, the rate of growth and the Euler-Poincaré characteristic. In particular, what influence does the deficiency have on the structure of an infinite group?

As an answer to the second problem, we will show how to construct the deficiency volume $V_{d}(G)$, which will imply the following

Theorem 1. Let $G$ be a f.p. group with $\operatorname{def}(G) \geq 2$. Then any two isomorphic finite-index subgroups of $G$ have the same index.

We will then introduce the rank volume $V_{r}(G)$ and will prove that $V_{r}(G)>0$ if $\operatorname{def}(G) \geq 2$. We will show that if $\Gamma$ maps onto $\Pi$, then $V_{r}(\Gamma) \geq V_{r}(\Pi)$, and derive the following corollary.

Corollary. Let $M$ be a compact manifold with residually finite fundamental group of deficiency $\geq 2$. Then any nonzero degree map $f: M \rightarrow M$ induces an automorphism of $\pi_{1}(M)$.

We introduce then our second main tool, the volume of a representation, as presented in [13] and [14]. For $\Gamma=\pi_{1}(M)$ where $M$ is a closed $n$-dimensional manifold, and a representation $\rho$ of $\Gamma$ in a real semi-simple Lie group $G, \operatorname{Vol}(G)=\left(\rho^{*}\left(B o r_{n}\right),[M]\right)$, where $[M] \in H_{n}(\Gamma, \mathbb{Z})$ is an image of the fundamental class of $M$ and $\operatorname{Bor}_{n} \in H_{\text {cont }}^{n}(G)$ is a Borel generator for the continuous cohomology. For example, if $G=S L_{2}(\mathbb{C})$ then $\operatorname{Vol}(\rho)$

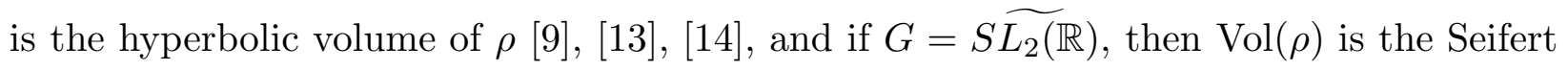
volume or a Chern-Simons invariant $[2,3]$. We set $\overline{V_{G}}(\Gamma)=\max _{\rho} \operatorname{Vol}(\rho)$; this is an lower volume. Observe that the maximum is finite by Cheeger-Simons rigidity [6], [14]. If $G=S O(n, 1)$ and $\Gamma=\pi_{1}(M)$, then using ideas of Gromov and Thurston, one shows [14] $\bar{V}_{G}(\Gamma) \leq \mu_{n} \cdot\|M\|$; it follows that $\bar{V}_{G}(\Gamma)$ gives arise to a volume in that case.

Let $\Gamma$ be a uniform lattice in $G$, then $\bar{V}_{G}(\Gamma) \geq \operatorname{Vol}(\Gamma \backslash G / K)>0$. This implies a complete higher-dimensional analogue of Gromov-Thurston Theorem [10]:

Theorem 2. Let $M=\Gamma \backslash G / K$ be a locally homogeneous manifold. For any $N$, there are only finitely many $d \in \mathbb{N}$, which are degrees of maps $f: N \rightarrow M$. In particular, any map $f: M \rightarrow M$ has degree 0 or \pm 1 . 
In case $G=S L_{2}(\mathbb{C})$ this is the Gromov-Thurston theorem. $\left.G=S \widetilde{(2,} \mathbb{R}\right)$ yields all Seifert fibrations, the main "complementary class" to hyperbolic manifolds according to Thurston's Geometrization Conjecture.

In section 3 we discuss the relation between $V_{S \widetilde{L(2, \mathbb{R})}}(\Gamma)$ and the generalized Hopf invariant.We then reformulate this connection combinatirially, in spirit with Quillen-Sullivan theory, which enables us to establish an effective estimate of the Chern-Simons invariant in terms of the number of simplices of a given triangulation of $M$, a "combinatorial complexity" of $M$. As a result, we get an explicit bound for the growth of topological complexity for finite converings of $M$ :

Theorem 3. Let $M$ be a plumbing of Seifert fibrations. Then the combinatorial complexity of a $d$-fold covering $N$ of $M$ grows at least as $(\log d)^{1-\varepsilon}, \varepsilon>0$.

I wish to thank a number of people for interesting discussion and useful information: Michel Boileau, Robert Brooks, Ilya Rips, Shicheng Wang, Alexander Lubotzky, Hyman Bass and Rostislav Grigorchuk. The work on the paper was initiated during my visits to Rühr-Universität Bochum and Université Paul Sabatier, Toulouse. I wish to emphasize that the treatment of Problem A is directly influenced by Gromov's work [9].

\section{Axioms fOR VOlumes:}

Let $\mathfrak{G}$ be the set of isomorphism classes of finitely-presented groups. A nonnegative function $\bar{V}: \mathfrak{G} \rightarrow \mathbb{R}$ is called an upper volume if for $\Delta \subset \Gamma$ a subgroup of finite index one has

$$
\bar{V}(\Delta) \leq(\Gamma: \Delta) \bar{V}(\Gamma) .
$$

Similarly, a lower volume is a function yielding the opposite inequality. A volume is a function for which an equality always holds.

Let $V: \mathfrak{G} \rightarrow \mathbb{R}$ be a volume. A group $\Gamma$ is call distinctable by $V$ if $V(\Gamma)$ is positive. The importance of this notion is seen form the following proposition.

1.1. Proposition. Let $V$ be a volume function and let $\Gamma$ be distinctable by $V$. Then any two isomorphic finite-index subgroups of $\Gamma$ have the same index.

Let $V$ be a volume. We say that $V$ is Hopfian, if for any epimorphism $\Gamma \rightarrow \Pi$ one has $V(\Pi) \leq V(\Gamma)$. 
1.2. Proposition. Let $V$ be a Hopfian volume, and let $\Gamma$ be distinctable by $V$. Then the image of any endomorphism $\varphi: \Gamma \rightarrow \Gamma$ is either all of $\Gamma$, or of infinite index in $\Gamma$. If $\Gamma$ is residually finite, then $\varphi$ is an isomorphism in the first case.

Proof: Let $\Pi=\varphi(\Gamma)$ and suppose $K(\Gamma: \Pi)<\infty$. Then $V(\Gamma) \geq V(\Pi)=(\Gamma: \Pi) V(\Gamma)>$ $V(\Gamma)$ a contradiction. So either $\Pi=\Gamma$ or $(\Gamma: \Pi)=\infty$. Since any residually finite group is Hopfian, the second statement also holds.

1.3: Let $\underline{V}$ be a lower volume, $\bar{W}$ is an upper volume and $V \leq W$. Define

$$
V(\Gamma)=\varliminf_{\Delta \rightarrow 1} \frac{\underline{V}(\Delta)}{[\Gamma: \Delta]} \quad, \bar{W}(\Gamma)=\varlimsup_{\Delta \rightarrow 1} \frac{\bar{W}(\Delta)}{(\Gamma: \Delta)}
$$

where $\underline{\text { lim}}$, are taken over the lattice of finite index subgroups. We claim

Proposition 1.3. $V$ and $W$ are volumes and $V \leq W$.

Proof: One checks immediately that $0 \leq V(\Gamma) \leq W(\Gamma)<\bar{W}(\Gamma)<\infty$. Since for $\Pi \leq \Delta \leq \Gamma, \quad \frac{V(\Pi)}{[\Delta: \Pi]}=[\Gamma: \Delta] \frac{V(\Pi)}{[\Gamma: \Pi)}, \quad V$ is volume and similarly for $W$.

1.4 Example: Define $V_{\chi}(\Pi)=\left\{\begin{array}{ll}|\chi(\Gamma)| & \text { if } \Gamma \text { is } F P \\ 0 & \text { otherwise. }\end{array}\right.$ Then $V_{\chi}$ is a volume.

Proof: Follows form [17], [7].

More interesting examples are given in the next section.

2. Deficiency and rank volumes. Let $\operatorname{def}_{+}(\Gamma)=\max (\operatorname{def}(\Gamma), 1)$.

2.1: Let $\underline{V}_{d}(\Gamma)=\operatorname{def}_{+}(\Gamma)-1$ and $\bar{V}_{r}(\Gamma)=r(\Gamma)-1$. We claim that $\underline{V}, \bar{W}$ yield the conditions of the Proposition 1.3. Indeed, obviously $\underline{V}_{d}(\Gamma) \leq \bar{V}_{r}(\Gamma)$ since $\operatorname{def}(\Gamma) \leq r(\Gamma)$. Moreover, for $\Delta \leq \Gamma$ of index $d$ one has

$$
\begin{gathered}
r(\Delta)-1 \leq d(r(\Gamma)-1) \\
\operatorname{def}(\Delta)-1 \geq d(\operatorname{def}(\Gamma)-1)
\end{gathered}
$$

[11]. So $\underline{V}_{d}$ is a lower volume and $\bar{V}_{r}$ is an upper volume. By Proposition 1.3 we get volumes $V_{d}$ and $V_{r}$ satisfying

$$
\operatorname{def}_{+}(\Gamma)-1 \leq V_{d}(\Gamma) \leq V_{r}(\Gamma) \leq r(\Gamma)-1
$$


In particular, if $\operatorname{def}(\Gamma) \geq 2$, then $\Gamma$ is distinctable by $V_{d}$ and $V_{r}$. We are now in position to prove Theorem 1.

Proof of Theorem 1: Since $\Gamma$ is distinctable by $V_{d}$, this follows from Proposition 1.1. 2.2: $\underline{V}_{r}$ is Hopfian. We claim that $V_{r}$ is a Hopfian volume. By definition $V_{r}(\Gamma)=$ $\varlimsup_{\Delta \rightarrow 0} \frac{\bar{V}_{r}(\Delta)}{[\Gamma: \Delta]}$. Let $\Gamma \stackrel{\varphi}{\rightarrow} \Pi$ be onto and let $\Lambda \leq \Pi$ be of index $d$. Then put $\Delta=\varphi^{-1}(\Lambda)$ so that $[\Gamma: \Delta]=d$ and $\left.\varphi\right|_{\Delta}: \Delta \rightarrow \Lambda$ is onto. Since $r(\Lambda) \leq r(\Delta)$ we get $V_{r}(\Gamma) \geq V_{r}(\Pi)$.

Corollary. Let $M$ be a compact manifold with residually finite fundamental group of deficiency $\geq 2$. Then any nonzero degree map $f: M \rightarrow M$ induces an automorphism of $\pi_{1}(M)$.

Proof: Since $\operatorname{deg} f \neq 0$, the image $f_{*}\left(\pi_{1}(M)\right)$ is of finite index in $\pi_{1}(M)$, by the standard argument. Then Proposition 1.2 implies that $f_{*}$ is an isomorphism.

3. Representation and volumes. Let $G$ be real semisimple Lie group. Let $K$ be the maximal compact subgroup of $G$ and let $X^{n}=G / K$. Fix a $G$-invariant volume form $\omega$ an $X$. By Borel, see e.g. [14] one has an element $\operatorname{Bor}(\omega) \in H_{\text {Cont }}^{n}(G)$. If $\Gamma \stackrel{\rho}{\rightarrow} G$ is a representation one has a map $B \Gamma \rightarrow B G^{\delta}$ and an induced map $H^{*}\left(G^{\delta}, \mathbb{R}\right) \rightarrow H^{*}(\Gamma, \mathbb{R})$. Composing with a natural map $H_{\text {Cont }}^{n}(G) \rightarrow H^{n}\left(G^{\delta}, \mathbb{R}\right)$ one gets an element Bor $(\rho, \omega) \in$ $H^{n}(\Gamma, \mathbb{R})$. We refer to [14] for direct geometric construction of this element and numerous applications.

Suppose we have a closed oriented manifold $M^{n}$ which is $K(\Gamma, 1)$. Then there is an element $[M] \in H_{n}(\Gamma, \mathbb{Z})$ and in fact $H_{n}(\Gamma, \mathbb{Z}) \approx \mathbb{Z}$ is generated by it. We put

$$
\operatorname{Vol}(\rho)=(\operatorname{Bor}(\rho, \omega),[M]) \in \mathbb{R}
$$

Now, we put

$$
V_{G}(\Gamma)=\max _{\rho}|\operatorname{Vol}(\rho)|
$$

One needs an argument to show that the maximum is finite. In fact, the Cheeger-Simons rigidity [6] implies that $\operatorname{Vol}(\cdot)$ is a locally constant function on representation variety $\operatorname{Hom}(\Gamma, G)$. On the other hand, the latter has finitely many components [9]. So $V_{G}(\Gamma)$ is finite. 
Let $\Delta<\Gamma$ be of finite index and let $N \rightarrow M$ be the corresponding covering. The diagram

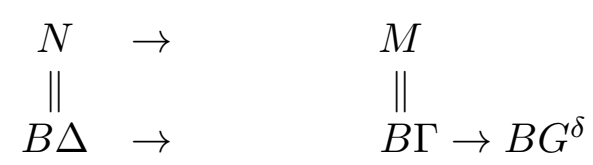

shows that $V_{G}(\Delta) \geq(\Gamma: \Delta) V_{G}(\Gamma)$. That is, $V_{G}(\cdot)$ is a lower volume. Moreover, let $Q$ be any closed manifold and let $f: Q \rightarrow M$ be a continuous map. Then

$$
V_{G}\left(\pi_{1}(Q)\right) \geq \operatorname{deg} f \cdot V_{G}(\Gamma)
$$

3.2: Now, let $\Gamma$ be a torsion-free uniform lattice in $G$. Then $[14] V_{G}(\Gamma) \geq \operatorname{mes}(\Gamma \backslash G / K)$, where the measure is induced by $\omega$. In particular, $V_{G}(\Gamma)$ is positive.

Now we are ready to prove

Theorem 2. Let $M=\Gamma \backslash G / K$ be a locally homogeneous compact manifold. For any compact oriented $Q, \operatorname{dim} Q=\operatorname{dim} M$, there are only finitely many $d \in \mathbb{Z}$ which are degrees of many $f: Q \rightarrow M$. In particular, any selfmap $f: M \rightarrow M$ has degree 0 or \pm 1 .

Proof: The inequality above implies

$$
\operatorname{deg} f \leq \frac{V_{G}\left(\pi_{1}(Q)\right)}{V_{G}(\Gamma)}
$$

and since $V_{G}(\Gamma)>0$, the RHS is finite. The second statement follows by application of the first statement to iterations of $f$, as usual.

EXAmPLE: Let $G=S O(n, 1)$ so that $M$ is compact hyperbolic. We claim that $V_{G}(\Gamma)$ is just a hyperbolic volume of $M$. Indeed, for any representation $\rho$ we have

$$
\operatorname{Vol}(\rho) \leq \operatorname{Vol}\left(\rho_{0}\right)=\operatorname{Vol}(M)
$$

where $\rho_{0}$ is the natural representation $([14])$.

In general, for any $M$ one has $V_{G}\left(\pi_{1}(M)\right) \leq \mu_{n}\|M\|$ where $\|M\|$ is Gromov's simplicial volume and $\mu_{n}$ is the Milnor constant. [14]

3.3.: Now let $G=S \widetilde{S L_{2}(\mathbb{R})}$ and let $M^{3}$ be a rational homology sphere. Let $\Gamma=\pi_{1}(M)$ and let $\rho: \Gamma \rightarrow S \widetilde{L_{2}(\mathbb{R})}$ be a representation. We wish to interpret Vol $(\rho)$ as a generalized Hopf 
invariant. Recall that $\widetilde{S L_{2}(\mathbb{R})}$ acts on the hyperbolic plane $\mathfrak{H}^{2}$. Let $V$ be the area form on $\mathfrak{H}^{2}$. Form a flat fiber bundle

$$
F=\tilde{M} \underset{\pi_{1}(M)}{\times} \mathfrak{H}^{2}
$$

it carries a self-parallel fiber-like two-form $\nu$.

This extends naturally to a closed two-form an $F$, using the flat connection; we keep the notation $\nu$ for this form [14]. Now, for any smooth section $s$ of $F \rightarrow M$ put $\lambda=s^{*} \nu$. This is a closed two-form on $M$. Since $M$ is a rational homology sphere, $\lambda=d \kappa$ for some $\kappa \in \Omega^{1}(M)$. Finally, compute $H(s)=\int_{M} \kappa \cdot \lambda$. We claim that independently on $s, H(X)=\operatorname{Vol}(\rho)$. Indeed, since $\mathfrak{H}^{2}$ is contractible, all sections are homotopic. Now the standard proof of the homotopy-invariance of Hopf invariant (in Whitehead form, see [8]) applies in our situation and shows that $H(s)$ is independent on $s$. Next, consider the diagram

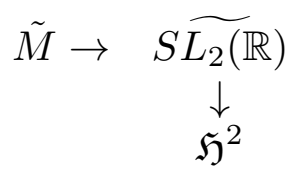

where the horizontal map $\varphi$ is any equivariant smooth map. For any left-invariant $\beta$ such that $d \beta=\pi^{*} \nu$ we have $\beta \cdot \pi^{*} \nu=\omega$. Let $s$ be a section of $\mathcal{F}$, coming form $\varphi$, then obviously $H(s)=\operatorname{Vol}(\rho)$.

Now, consider a triangulation of $M$ and let $\gamma$ be a simplicial two-cochain, given by integration of $s^{*} \nu$ over simplices. We can compute $H(s)$ combinatorially, finding onecochain $\alpha$ such that $d \alpha=\gamma$ and then computing $(\alpha \cup \gamma,[M])$. Let $s$ be a section, given by Thurston straightening technique $([14])$. Then $(\gamma, \sigma) \leq \pi$ for any two-simplex $\sigma$.

The map $d+\delta: C^{1}(M, \mathbb{R}) \oplus \tilde{C}^{3}(M, \mathbb{R}) \rightarrow \tilde{C}^{0}(M, \mathbb{R}) \oplus C^{2}(M, \mathbb{R})$ is an isomorphism since $M$ is a rational homology sphere. Let $c_{i}=\operatorname{dim} C^{i}$ be a number of $i$-simplices. The matrix of $d+\delta$ is a $\left(c_{1}+c_{3}-1\right) \times\left(c_{1}+c_{3}-1\right)$ integer invertible matrix, with all entries zero or one and the number of nonzero entries in any column or row does not exceed the adjacency at the triangulation (the number of simplices of the next dimension, adjacent to a given one), say $a$. So the sup-norm of $(d+\delta)^{-1}(\gamma)$ is bounded by $(\sqrt{a})^{c_{1}+c_{3}-1}\left(c_{1}+c_{3}-1\right) \cdot \pi$, by Hadamard inequality. It follows that

$$
|\operatorname{Vol}(\rho)|=|(\alpha \cup \gamma,[M])| \leq(\sqrt{a})^{c_{1}+c_{3}-1}\left(c_{1}+c_{3}-1\right) \cdot \pi^{2} \cdot c_{3} .
$$


Now, let $\varepsilon: N \rightarrow M$ be a $d$-sheeted covering. For any triangulation of $N$ we have

$$
d|\operatorname{Vol}(\rho)| \leq\left(\sqrt{a^{\prime}}\right)^{c_{1}^{\prime}+c_{3}^{\prime}-1}\left(c_{1}^{\prime}+c_{3}^{\prime}-1\right) \pi^{2} c_{3}^{\prime}
$$

or, assuming $\operatorname{Vol}(\rho)>0, \frac{1}{2}\left(c_{1}^{\prime}+c_{3}^{\prime}-1\right) \log a^{\prime} \geq \log d+\operatorname{const}(M)+O\left(\log \left(c_{1}^{\prime}+c_{3}^{\prime}\right)\right)$. Now $c_{1}^{\prime} \leq 2 c_{3}^{\prime}$ and $a^{\prime} \leq c_{3}^{\prime}$. So

$$
\left.\frac{3}{2} c_{3}^{\prime} \log c_{3}^{\prime} \geq \log d+\operatorname{const}(m)+O\left(\log c_{3}^{\prime}\right)\right)
$$

Summing up, we have

THEOREM 3. . Let $M$ be a geometric non-hyperbolic homology sphere (i.e. a plumbing of Seifert fibrations). Then the topological complexity of its $d$-sheeted coverings grows at least as $(\log d)^{1-\varepsilon}$, for any $\varepsilon>0$.

Proof: Any such $M$ admits a representation

$$
\rho: \pi_{1}(M) \rightarrow \widetilde{S L_{2}(\mathbb{R})}
$$

with $\operatorname{Vol}(\rho) \neq 0$.

\section{Concluding remarks.}

4.1. VOLUMES FOR PRO- $p$ GROUPS: Let $G$ be a pro- $p$ group with finitely generated homology groups. Then $\operatorname{dim}_{\mathbb{F}_{p}}\left(H^{k}\left(G, \mathbb{F}_{p}\right)\right)$ is a lower volume, that is, for $H<G$ an open subgroup.

$$
\left.\operatorname{dim}_{\mathbb{F}_{p}} H^{k}\left(H, \mathbb{F}_{p}\right) \leq[G: H] \operatorname{dim}_{\mathbb{F}_{p}} H^{k}\left(G, \mathbb{F}_{p}\right)\right)
$$

Therefore $\mu_{k}(G)=\varlimsup_{H<G} \frac{\operatorname{dim} H^{k}\left(H, \mathbb{F}_{p}\right)}{[G: H]}$ is a finite volume. If $\operatorname{dim}_{\mathbb{F}_{p}} H^{1}(G)-\operatorname{dim}_{\mathbb{F}_{p}} H^{2}(G) \geq 2$, then $\mu_{1}(G)>0$. We refer to [15] for details.

\section{References}

[1] H. Bass, Euler characteristic and characters of discrete groups. Inv. Math., 28 (1976), 351-342.

[2] R. Brooks, W. Goldman, Volumes in Seifert space, Duke Math. J., 51 (1984), 529-545.

[3] R. Brooks, W. Goldman, The Godbillon-Wey invariant of a transversely homogeneous foliation, TAMS 286 (1984), 651-664. 
[4] K. Brown, Euler characteristics of discrete groups and $G$-spaces, Inv. Math. 27 (1974), 229-264.

[5] J. Carlson, D. Toledo, Harmonic mappings of Kähler manifolds to locally symmetric spaces, Publ. Math. IMES, 69 (1989), 173-201.

[6] J. Cheeger, J. Simons, Differential characters and geometric invariants, in Geometry and topology, J. Alexander and J. Karer, eds, LNM 1167, Springer, 1985, 50-80.

[7] I. Chiswell, Euler characterics of Groups, Math. Z., 147 (1976), 1-11.

[8] E.Friedlender,P. Griffiths, J. Morgan, Homotopy theory and differential forms, Mimeographed notes (1972).

[9] M. Gromov, Volume and bounded cohomology, Publ. Math. IHES, 56 (1983), 5-99.

[10] M. Gromov, Hyperbolic manifolds according to Thurston and Jorgensen, Sem. Bourbaki 1979/1980, 40-53.

[11] A. Lubotzky, Group presentation, $p$-adic analytic groups and lattices in $S L(2, \mathbb{C})$, Annals of Mathematics, 118 (1983), 115-130.

[12] R. Lyndon, Problems in combinatorial group theory, Annals of Math. Stud. 111 (1987), 3-33.

[13] A. Reznikov, Harmonic maps, hyperbolic cohomology and higher Milnor inequalities, Topology, 32 (1993), 899-907.

[14] A. Reznikov, Rationality of secondary classes, J. Diff. Geom., to appear.

[15] A. Reznikov, A spectral sequence for pro- $p$ group cohomology and Adem inequalities, in preparation.

[16] J.Stallings,An extension theorem for Euler characteristics of groups, preprint (1976).

[17] C. T. C. Wall, Rational Euler characteristics, Proc. Cam. Phil. Soc. 57 (1961), $182-183$.

[18] S. Wang, Y. Q. Wu, Covering invariants and co-Hopficity of 3-manifold groups, Proc. LMS, 68 (1994), 203-224.

Institute of Mathematics

The Hebrew University

Givat Ram 91904, Jerusalem 
e-mail: simplex@math.huji.ac.il

and

Max-Planck-Institut für Mathematik

e-mail:reznikov@mpim-bonn.mpg.de 